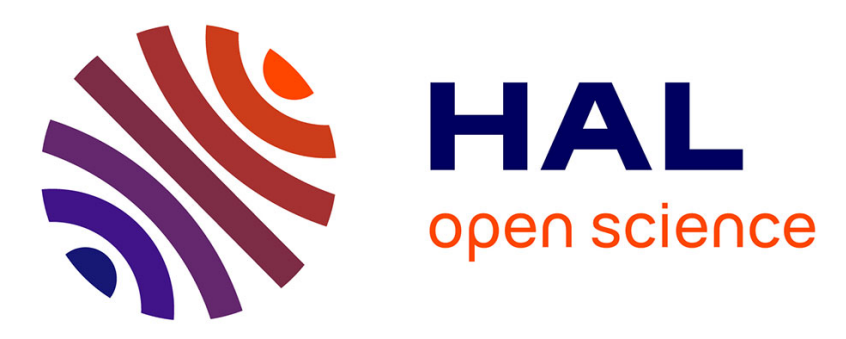

\title{
Pushing the limit of lithography for patterning two-dimensional lattices in III-V semiconductor quantum wells
}

\author{
Nathali Alexandra Franchina Vergel, C. Post, Francois Vaurette, Yannick \\ Lambert, Dmitri Yarekha, Christophe Coinon, G. Fleury, T.S. Kulmala, T.
} $\mathrm{Xu}, \mathrm{L}$. Desplanque, et al.

\section{To cite this version:}

Nathali Alexandra Franchina Vergel, C. Post, Francois Vaurette, Yannick Lambert, Dmitri Yarekha, et al.. Pushing the limit of lithography for patterning two-dimensional lattices in III-V semiconductor quantum wells. 5th IEEE Electron Devices Technology \& Manufacturing Conference, EDTM 2021, Apr 2021, Chengdu, China. pp.1-3, 10.1109/EDTM50988.2021.9420884 . hal-03261329

\section{HAL Id: hal-03261329 \\ https://hal.science/hal-03261329}

Submitted on 5 Nov 2021

HAL is a multi-disciplinary open access archive for the deposit and dissemination of scientific research documents, whether they are published or not. The documents may come from teaching and research institutions in France or abroad, or from public or private research centers.
L'archive ouverte pluridisciplinaire HAL, est destinée au dépôt et à la diffusion de documents scientifiques de niveau recherche, publiés ou non, émanant des établissements d'enseignement et de recherche français ou étrangers, des laboratoires publics ou privés.

$$
\text { Copyright }
$$




\title{
Pushing the limit of lithography for patterning two-dimensional lattices in III-V semiconductor quantum wells
}

\author{
N.A. Franchina Vergel ${ }^{1}$, C. Post ${ }^{2}$, F. Vaurette ${ }^{1}$, Y. Lambert ${ }^{1}$, D. Yarekha ${ }^{1}$, C. Coinon ${ }^{1}$, G. Fleury ${ }^{3}$, T. \\ Kulmala $^{4}, \mathrm{~T} . \mathrm{Xu}^{5}$, L. Desplanque ${ }^{1}, \mathrm{X}$. Wallart ${ }^{1}, \mathrm{D}$. Vanmaekelbergh ${ }^{2}$, C. Delerue ${ }^{1}$, B. Grandidier ${ }^{1}$ \\ ${ }^{1}$ Univ. Lille, CNRS, Centrale Lille, Univ. Polytechnique Hauts-de-France, ISEN-Yncrea Hauts-de-France, UMR 8520 \\ - IEMN, 59000 Lille, France, bruno.grandidier@isen.iemn.univ-lille1.fr \\ ${ }^{2}$ Debye Institute for Nanomaterials Science, Utrecht University, The Netherlands, ${ }^{3}$ Univ. Bordeaux, CNRS, Bordeaux \\ INP, LCPO, UMR 5629, F-33600 Pessac, ${ }^{4}$ SwissLitho AG, Technoparkstrasse 1, 8005 Zurich, Switzerland, ${ }^{5}$ School of \\ Mechatronic Engineering and Automation, Key Laboratory of Advanced Display and System Applications, Ministry \\ of Education, Shanghai University, 200072, Shanghai, China,
}

\begin{abstract}
Building two-dimensional lattices in semiconductor quantum-wells offers the prospect to design distinct energy-momentum dispersions, including conical intersections and nondispersive bands. Here, we compare three lithographic patterning methods, ebeam lithography, block copolymer lithography and thermal scanning probe lithography to produce a honeycomb lattice in an $\operatorname{In}_{0.53} \mathrm{Ga}_{0.47}$ As quantum well. We weigh up the pros and cons of each method to reach lattice constants smaller than $20 \mathrm{~nm}$ with a minimum of dispersion in the pore size.
\end{abstract}

(Keywords: Lithography, III-V semiconductors, two-dimansional lattices)

\section{Introduction}

Semiconductor heterostructures, including quantum wells, are essential components of the most advanced semiconductor devices. Their usefulness stems from the ability they provide to control the fundamental parameters of a semiconductor crystal: its band gap, the effective mass of the charge carriers, its refractive indice. Of particular interest is the possibility to locally modify the band structure of the semiconductor and so control the motion of the charge carriers. While these modifications have mainly been achieved along the growth direction of the heterostructures, there is a strong interest to further tailor the band structure of a semiconductor quantum-well $(\mathrm{QW})$ by modulating the potential through lateral confinement effects.

Beautiful examples of band structure engineering have been obtained by building two-dimensional (2D) artificial lattices in a 2D free electron gas at the surface of a metal. With the help of a tip, atoms and molecules have been manipulated in a scanning tunneling microscope to produce honeycomb or Lieb lattices, which gave rise to particular energymomentum dispersion including Dirac cones and flat bands $[1,2,3]$. As the band structure is determined by the symmetry of the lattice, identical band structures can in principle be created in a semiconductor QW by nanopatterning a 2D array of potential barriers at the surface or inside the QW. However, the formation of band with widths in the
$10 \mathrm{meV}$ range, compatible with applications in information technologies, imposes severe constraints on the electron confinement and on the coupling between the lattice sites. Despite promising results with honeycomb lattices produced in GaAs/AlGaAs heterostructures where the lattice constant was around $40 \mathrm{~nm}$ [4], the detection of the Dirac cone has remained elusive. Here, considering $\mathrm{In}_{0.53} \mathrm{Ga}_{0.47} \mathrm{As} / \mathrm{InP}$ heterostructures, we examine the performances of three different lithographic patterning methods to obtain smaller lattice constants, that are expected to produce wider energy-momentum dispersions.

\section{Experimental}

$\mathrm{In}_{0.53} \mathrm{Ga}_{0.47} \mathrm{As} / \mathrm{InP}$ heterostructures were grown by molecular beam epitaxy (MBE). To protect the surface of the QW from carbon contaminations, the QW was capped with $16 \mathrm{~nm}$ of $\mathrm{SiO}_{2}$ using plasmaenhanced chemical vapour deposition (PECVD). As shown in Fig. 1, nanopatterning was performed with three different lithographic methods: e-beam lithography (EBL), block copolymer lithography (BCPL) and thermal scanning probe lithography ( $t-$ SPL).

For the EBL method, the samples were dehydrated by heating the surface to $180{ }^{\circ} \mathrm{C}$ for 10 mins. A diluted polymethylmethacrylate (PMMA) $950 \mathrm{~K} 4 \%$ resist with a thickness of $50 \mathrm{~nm}$ was spin-coated on top of the sample, followed by an annealing step at $80{ }^{\circ} \mathrm{C}$ for $1 \mathrm{~min}$ on a hot plate and a bake out at 180 ${ }^{\circ} \mathrm{C}$ for 10 min. E-beam exposure was performed using a Raith EBPG 5000Plus equipment. The development of the PMMA resist was done by plunging the samples into a mixture of methyl isobutyl ketone (MIBK) and isopropyl alcohol IPA $(1: 2)$ for $60 \mathrm{~s}$. The samples were subsequently rinsed in IPA for $40 \mathrm{~s}$ to stop the reaction and dried under nitrogen flow.

The second method involved the self-assembly of a BCP on top of the $\mathrm{SiO}_{2}$ layer to generate a hexagonal triangular pattern. A poly(styrene)-blockpoly(methyl methacrylate) (PS- $b$-PMMA) copolymer was synthesized by the sequential anionic polymerization of styrene and methyl 
methacrylate leading to well-defined $\mathrm{BCP}$ architectures with low dispersity. The BCP was dissolved in toluene $(2 \mathrm{wt} \%)$ and spin-coated onto the $\mathrm{SiO}_{2}$ surface chemically-modified by grafting a poly(styrene)-stat-poly(methyl methacrylate) (PSstat-PMMA) brush to control the orientation of the BCP structure. The self-assembly of the BCP layer was further promoted by thermal annealing at 220 ${ }^{\circ} \mathrm{C}$ leading to out-of-plane PMMA cylinders packed into a hexagonal triangular lattice.

The third method relied on thermal scanning probe lithography (t-SPL) [5,6]. A four-layer pattern transfer stack (8-nm-thick polyphthalaldehyde (PPA) thermal resist layer / 2-nm-thick PMMA buffer layer / 1.5-nm-thick spin-coatable silicon hard mask ( $\mathrm{SH}$ 113, PiBond Oy) / 40-nm-thick organic transfer layer (OTL 405, PiBond Oy)), was spin-coated on the $\mathrm{SiO}_{2}$ layer. A commercial t-SPL system (NanoFrazor Explore, Heidelberg Instruments Nano) was used to pattern the PPA layer with a tip heater temperature of $700{ }^{\circ} \mathrm{C}$. Followed by a brief $\mathrm{O}_{2} / \mathrm{N}_{2}$ reactive ion etching descum step to remove the PMMA buffer layer at the bottom of the pattern, the PPA pattern was first transferred into the silicon hard mask in a $\mathrm{CHF}_{3}$ reactive ion etching (RIE) step and further into the OTL in another $\mathrm{O}_{2}$ RIE step.

\section{Results and discussion}

For each method, the same patterning was performed: a triangular antidot lattice to confine the electrons in a honeycomb geometry. Optimization of the parameters with EBL to obtain the minimum periodicity between the pores in the PMMA resist led to a periodicity of $39 \mathrm{~nm}$, as shown in the scanning electron micrograph of Fig. 2. Further adjustments of the e-beam parameters to reach a smaller periodicity of $30 \mathrm{~nm}$ did not allow to create a homogeneous array of pores. Due to proximity effects, a strong overlap of the pores occured. In parallel, the mask was prepared with a BCP layer, where the PMMA domains are selectively removed with plasma chemistry. This process resulted in the formation of a PS honeycomb mask. Another approach consisted in patterning pores into a PPA layer, which locally decomposes upon contact with the hot tip of an atomic force microscope. Both methods led to smaller periodicities. Figure 2 shows two examples where periodicities of $36 \mathrm{~nm}$ and 30 $\mathrm{nm}$ were achieved with BCPL and t-SPL respectively. Important to the design of distinct energy-momentum dispersions is the disorder that is caused by nanopatterning imperfections. Based on a statistical analysis of the pore sizes measured in SEM [7], we found a smaller disorder for the mask prepared with t-SPL in comparison with EBL and BCPL. Indeed, comparison of the full width at half maximum in the distribution of the pore sizes yields $1.0 \mathrm{~nm}, 1.4 \mathrm{~nm}$ and $1.6 \mathrm{~nm}$ for t-SPL, BCPL and EBL respectively.

In an attempt to further reduce the periodicity of the honeycomb lattices, masks with periodicities of 26 $\mathrm{nm}$ and $23 \mathrm{~nm}$ were achieved with t-SPL and BCPL respectively. At these dimensions, the pore size distribution obtained with t-SPL become broader than the one obtained with BCPL. As a result, the transfer of the triangular pattern to first the $\mathrm{SiO}_{2}$ layer and subsequently to the $\mathrm{In}_{0.53} \mathrm{Ga}_{0.47} \mathrm{As} \mathrm{QW}$ was performed from the BCP masks only. A mixture of $\mathrm{CHF}_{3}$ and $\mathrm{CF}_{4}$ was used for the reactive ion etching (RIE) of the $\mathrm{SiO}_{2}$ layer, whereas $\mathrm{BCl}_{3}$ based inductively coupled plasma (ICP) etching was selected for the processing of the pore in the QW. Fig. 3 reveals the homogeneous formation of pore in the QW at the end of the technological processes for a BCP mask with a periodicity of $36 \mathrm{~nm}$. Moreover, based on cross-sectional scanning transmission electron microscopy, the depth of the pore corresponds to the whole thickness of the QW, indicating the high quality of the transfer pattern. In contrast, a rather inhomogeneous lattice of pores was found for the BCP mask with a periodicity of 23 $\mathrm{nm}$, which was caused by an unsatisfactory etching of the $\mathrm{SiO}_{2}$ layer.

\section{Conclusion}

We have examined three lithographic techniques to create triangular antidot lattices in an $\mathrm{In}_{0.53} \mathrm{Ga}_{0.47} \mathrm{As}$ QW. We have shown that both t-SPL and BCPL allow to go beyond EBL, where electron scattering causes limiting proximity effects. A honeycomb lattice with a periodicity of $36 \mathrm{~nm}$ was successfully built using BCP. Smaller periodicities in the $20 \mathrm{~nm}$ range are within reach by strengthening the quality of the transfer pattern.

\section{Acknowledgments}

This work was supported by the French National Research Agency (ANR-16-CE24-0007-01 Dirac III-V), the RENATECH network, the H2020 program (ERC Advanced Grant 692691-“FIRST STEP"), the Dutch Research Council (NWO 
Chemistry - Toppunt "Superficial superstructures") and the Natural Science Foundation of Shanghai (19ZR1419500).

\section{References}

[1] K. K. Gomes, W. Mar, W. Ko, F. Guinea, H. C. Manoharan, Nature 483, 306-310, (2012).

[2] R. Drost, T. Ojanen, A. Harju, P. Liljeroth, Nat. Physics 13, 668-671 (2017).
[4] S. Wang et al., Nat. Nanotechnology 13, 29-33 (2018).

[5] S.T. Howell, A. Grushina, F. Holzner, J. Brugger, Microsyst. Nanoeng. 6, 21 (2020).

[6] Y. K. Ryu Cho et al., ACS Nano 11, 11890-11897 (2017).

[7] L. C. Post et al., Nanotechnology 30, 155301 (2019).

[3] M. R. Slot et al., Nat. Physics 13, 672-676 (2017).

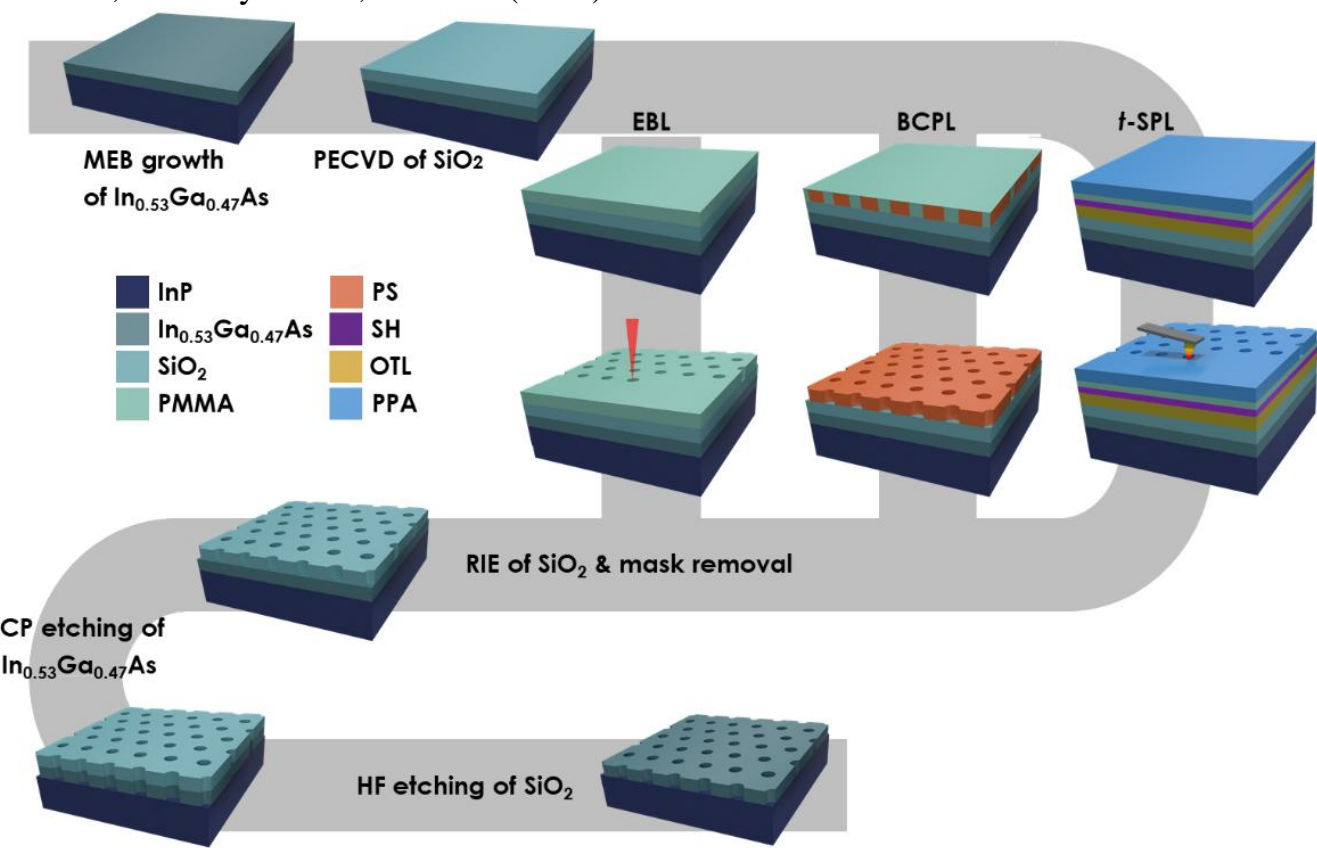

Fig. 1: Schematic workflow diagram for lithographic fabrication of a triangular antidot lattice in an $\operatorname{In}_{0.53} \mathrm{Ga}_{0.47} \mathrm{As}$ quantum well grown on a InP (001) substrate by molecular beam epitaxy (MBE). Three techniques were used: ebeam lithography (EBL), block copolymer lithography (BCPL) and thermal scanning probe lithography (t-SPL).

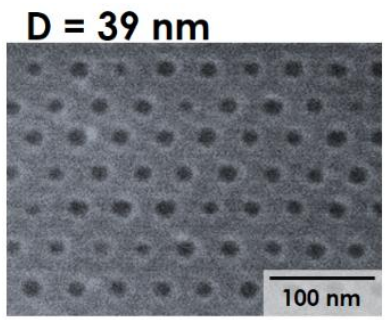

EBL

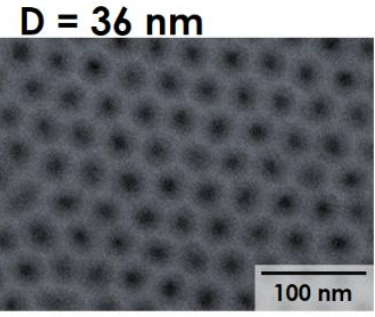

BCPL

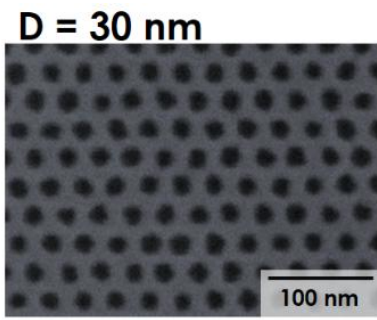

$t-S P L$

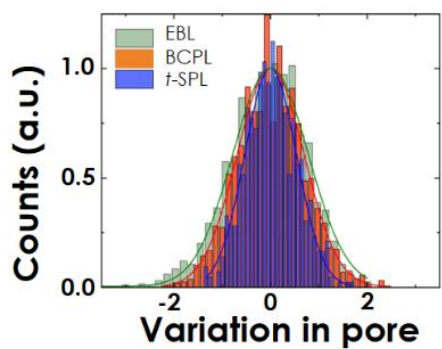

radius $(\mathrm{nm})$

Fig. 2: SEM images of the masks prepared with EBL, BCPL and t-SPL. The periodicity of the triangular lattice is indicated for each mask. Comparison of the pore size variation for the three different masks.
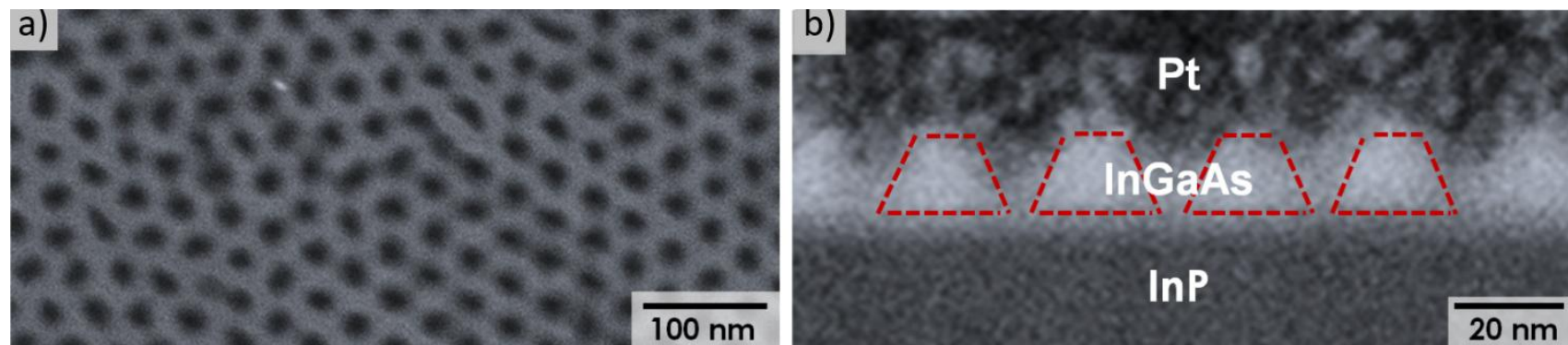

Fig. 3: a) SEM and b) cross-sectional TEM images of the honeycomb $\operatorname{In}_{0.53} \mathrm{Ga}_{0.47}$ As quantum well nanoperforated 
with a $36 \mathrm{~nm}$ periodicity from a $\mathrm{BCP}$ mask. 